\title{
Níveis de Lisina para Frangos de Corte de 22 a 40 e 42 a 48 dias de Idade 1
}

\author{
Walter Amaral Barboza², Horacio Santiago Rostagno ${ }^{3}$, Luiz Fernando Teixeira Albino³, \\ Paulo Borges Rodrigues ${ }^{4}$
}

\begin{abstract}
RESUMO - Foram realizados dois experimentos para determinar a exigência nutricional de lisina total para frangos de corte Hubbard e Ross de ambos os sexos, no período de 22 a 40 e de 42 a 48 dias de idade. Foram avaliados ganho de peso, consumo de ração, conversão alimentary e rendimento de peito com osso e carne de peito. Para cada fase, o delineamento experimental utilizado foi o de blocos casualizados, em esquema fatorial 2 × 2 × 6, constituídos de dois sexos, duas marcas e seis níveis dietéticos de lisina, com quatro repetições e um total de 1920 aves. Dietas basais com $0,80 \%$ de lisina, 19,8\% PB e $3100 \mathrm{kcal} \mathrm{EM/kg}$, no primeiro experimento, e $0,75 \%$ de lisina, $18,5 \% \mathrm{~PB}$ e $3200 \mathrm{kcal} \mathrm{EM} / \mathrm{kg}$, no segundo experimento, foram suplementadas com seis níveis de lisina $(0 ; 0,06 ; 0,12 ; 0,18 ; 0,24 ; \mathrm{e} 0,30 \%)$. Considerando-se ganho de peso, conversão alimentar, rendimentos de peito com osso e carne de peito dos frangos, as estimativas das exigências dietéticas de lisina total na fase de 22 a 40 dias foram 0,80\% para fêmeas Ross, 0,993\% para fêmeas Hubbard, 0,998\% para machos Hubbard e 1,03\% para machos Ross, respectivamente. Para a fase de 42 a 48 dias, recomenda-se o nível de 0,90\% de lisina total na ração para ambos os sexos das duas marcas comerciais.
\end{abstract}

Palavras-chave: exigência nutricional, frangos de corte, lisina total

\section{Lysine Levels for Broiler Chickens from 22 to 40 and 42 to 48 Days of Age}

\begin{abstract}
Two experiments were carried out to determine the nutritional requirement of total lysine for Hubbard and Ross broiler chickens of both sexes, from 22 to 40 and from 42 to 48 days of age. Weight gain, feed intake, feed:gain ratio, breast with bone yield, and breast meat yield were evaluated. In each period, a completely randomized block experiment design in a factorial $2 \times 2 \times 6$ was used, with two sexes, two trademarks and six dietary lysine levels, with four replicates using a total of 1920 birds. The basal diets with $0.80 \%$ of lysine, $19.8 \% \mathrm{CP}$ and $3100 \mathrm{kcal} \mathrm{ME} / \mathrm{kg}$ in the first experiment and $0.75 \%$ lysine, $18.5 \% \mathrm{CP}$ and $3200 \mathrm{kcal} \mathrm{ME} / \mathrm{kg}$ in the second experiment, were supplemented with six lysine levels $(0,0.06,0.12,0.18,0.24$ and $0.30 \%)$. Considering weight gain, feed:gain ratio, breast with bone yield and breast meat yield of the chickens, the total lysine dietary requirement estimates from 22 to 40 days were $0.80 \%$ for females Ross, $0.993 \%$ for female Hubbard, $0.998 \%$ for males Hubbard and $1.03 \%$ for males Ross, respectively. For the period from 42 to 48 days, the dietary total lysine level recommended was $0.90 \%$ for both sexes and trade marks.
\end{abstract}

Key Words: broiler chickens, nutritional requirement, total lysine

\section{Introdução}

O progresso genético na taxa de crescimento, na conversão alimentar e no rendimento de carcaça é desafio constante do ponto de vista nutricional. Uma vez que a expressão fenotípica do potencial genético depende do ambiente e da nutrição, é importante conhecer as exigências nutricionais das aves em processo de melhoramento, para se obter o máximo desempenho das mesmas.

A suplementação das rações comerciais com aminoácidos sintéticos tem proporcionado facilidades no ajuste das formulações destas rações, possibilitando a obtenção dos níveis exigidos de aminoácidos essenciais. Para a elaboração de um programa nutricional, é comum o nutricionista basear-se nas recomendações das tabelas publicadas nos Estados Unidos (SCOTT et al. 1982; WALDROUP 1991; NATIONAL RESEARCH COUNCIL - NRC 1994), na Europa (AGRICULTURAL RESEARCH COUNCIL - ARC, 1975; INSTITUT NATIONAL DE LA RECHERCHE AGRONOMIQUE - INRA, 1984; e AEC, 1987) e no Brasil (ROSTAGNO et al., 1983; ROSTAGNO, 1990) ou nas recomendações dos manuais de alimentação e manejo das marcas comerciais fornecidos pelos detentores do material genético. Entretanto, os dados confundem-se graças às interações que ocorrem entre os diversos fatores ambientais envolvidos. A diversidade de informações exige cautela na análise dos objetivos e das particu-

\footnotetext{
1 Parte da tese apresentada à Universidade Federal de Viçosa para a obtenção do título de "Doctor Science".

2 Professor do Departamento de Zootecnia da UFRPE - Recife, PE.

3 Professor do Departamento de Zootecnia da UFV - Viçosa, MG

4 Professor do Departamento de Zootecnia da UFLA - Lavras, MG.
} 
laridades em que os dados de cada trabalho são coletados. Em geral, verificam-se consideráveis variações quanto aos valores de exigência de metionina (JENSEN et al., 1989; HICKLING et al., 1990) e lisina (AL-NASSER et al., 1986) para frangos de corte. Por isso, a determinação das exigências nutricionais destes aminoácidos para frangos de corte ainda é fator de grande importância para a moderna avicultura industrial.

REZENDE et al. (1980a), trabalhando com frangos de corte, Hubbard, sob altas temperaturas, no período de 29 a 42 dias de idade, verificaram exigência de $0,75 \%$ de lisina nas rações com $16 \%$ de proteína bruta. Entretanto, Uzu et al. (1989), citados por ACAR et al. (1991), estimaram exigência de 0,90\% de lisina na ração para frangos de corte na fase de 22 a 49 dias de idade.

MORAN JR. e BILGILI (1990) relataram que é possível melhorar o rendimento dos cortes nobres de frangos, pois, trabalhando com machos e fêmeas de diferentes marcas comerciais, no período de 28 a 42 dias de idade, observaram que, ao se elevar o teor de lisina da dieta, o ganho de peso das aves não melhorou, mas houve aumento significativo no rendimento de peito e coxas e decréscimo no percentual de gordura da carcaça. HICKLING et al. (1990) verificaram que ganho de 15 a $20 \mathrm{~g}$ na carne de peito pode ser obtido pela suplementação de metionina e lisina em $12 \%$ acima do recomendado pelo NRC (1984). Os resultados são de importância fundamental, visto que o comércio do frango inteiro não é mais exclusivo, com tendência crescente à preferência por carne na forma de cortes (DUDLEY- CASH, 1992).

As recomendações dos níveis dietéticos de lisina, para frangos de corte na fase de crescimento (29 a 42 dias), segundo ROSTAGNO (1990) e NRC (1994), na fase de 22 a 42 dias, com $3200 \mathrm{kcal}$ de EM/kg de ração, é de 0,995 e $1,0 \%$, respectivamente.

Dessa forma, o objetivo deste trabalho foi avaliar a exigência nutricional de lisina para machos e fêmeas de duas marcas comerciais, nos períodos de 22 a 40 e de 42 a 48 dias de idade.

\section{Material e Métodos}

Os experimentos foram conduzidos no setor de Avicultura do Departamento de Zootecnia, do Centro de Ciências Agrárias da Universidade Federal de Viçosa. Para cada fase, foram utilizados 1920 frangos de corte, em sexos separados, das marcas comerciais Hubbard e Ross, em um delineamento experimental em blocos casualizados, em esquema fatorial $2 \times 2 \times 6$, constituído de dois sexos, duas marcas e seis tratamentos (seis níveis de lisina), com quatro repetições por tratamento e 20 aves por unidade experimental.

Os animais foram submetidos a duas dietas basais (Tabela 1), calculadas para atender às exigências nutricionais preconizadas por ROSTAGNO et al. (1990), exceto para lisina, formuladas à base de milho e farelo de soja e suplementadas com seis níveis de lisina $(0 ; 0,06 ; 0,12 ; 0,18 ; 0,24 ; \mathrm{e} 0,30 \%)$ sob a forma de L-Lis.HCl 98\% (78,4\% de lisina), em substituição ao amido de milho, correspondendo aos níveis de 0,8 ; 0,$86 ; 0,92 ; 0,98 ; 1,04 ;$ e $1,10 \%$ e 0,$75 ; 0,81 ; 0,87 ; 0,93$; 0,99 ; e $1,05 \%$ de lisina total nas dietas para as fases de 22 a 40 e de 42 a 48 dias, respectivamente. Para a fase de 22 a 40 dias, o peso inicial da aves da marca comercial Hubbard foi de 763,8 e 698,2 g para machos e fêmeas, respectivamente, e para a marca comercial Ross, de 720,0 e de 649,6 g para machos e fêmeas, respectivamente. Para a fase de 42 a 48 dias, o peso médio inicial das aves da marca comercial Hubbard foi de 1886,7 e 1732,5 g para machos e fêmeas, respectivamente, e para a marca comercial Ross, de 1850,0 e 1657,2 g, para machos e fêmeas, respectivamente.

Ao final de cada fase, foram avaliados consumo de ração, ganho de peso (GP) e conversão alimentar (CA) e retiradas 10 aves, ao acaso, de cada unidade experimental, para avaliar o rendimento de peito com osso e carne de peito.

As análises estatísticas desses parâmetros foram realizadas de acordo com o programa Sistema para Análises Estatísticas e Genética - SAEG, de acordo com EUCLYDES (1983), e a estimativa das exigências em lisina, estabelecida por meio dos modelos de regressão linear e quadrático e do modelo descontínuo LRP - Linear Response Plateau (BRAGA, 1983), considerando-se na escolha do modelo, uma vez respeitada a interpretação biológica, a menor soma de quadrados.

\section{Resultados e Discussão}

Níveis de lisina da ração no desempenho de frangos de corte no período de 22 a 40 dias de idade

Conforme se observa na Tabela 2 , na qual se encontram os dados referentes ao desempenho das aves no período de 22 a 40 dias, com exceção do consumo de ração, para o qual não houve efeito significativo ( $P>0,05)$, e da conversão alimentar das 
Tabela 1 - Composição percentual da dieta basal

Table 1 - Percentage composition of the basal diet

\begin{tabular}{|c|c|c|}
\hline \multirow[t]{2}{*}{$\begin{array}{l}\text { Ingrediente (\%) } \\
\text { Ingredient }\end{array}$} & \multicolumn{2}{|c|}{$\begin{array}{l}\text { Período } \\
\text { Period }\end{array}$} \\
\hline & $22-40$ dias & $42-48$ dias \\
\hline Milho & 52,548 & 53,958 \\
\hline Corn & & \\
\hline Sorgo & 15,000 & 15,000 \\
\hline Sorghum & & \\
\hline $\begin{array}{l}\text { Farelo de soja, } 46 \% \\
\text { Soybean meal, } 46 \%\end{array}$ & 19,901 & 18,620 \\
\hline $\begin{array}{l}\text { Farelo de glúten de milho } \\
\text { Corn gluten meal }\end{array}$ & 7,649 & 6,000 \\
\hline $\begin{array}{l}\text { Óleo de soja } \\
\text { Soybean oil }\end{array}$ & 0,869 & 2,480 \\
\hline Fosfato bicálcico & 1,707 & 1,642 \\
\hline Cálcario & 1,110 & 1,137 \\
\hline $\begin{array}{l}\text { Limestone } \\
\text { Sal (Salt) }\end{array}$ & 0,300 & 0,300 \\
\hline $\begin{array}{l}\text { DL-Metionina, } 99 \% \\
\text { DL-Methionine }\end{array}$ & 0,146 & 0,133 \\
\hline Amido (Starch) & 0,400 & 0,400 \\
\hline $\begin{array}{l}\text { Premix mineral } \\
\text { Mineral premix }\end{array}$ & 0,050 & 0,050 \\
\hline $\begin{array}{l}\text { Premix vitamínico } \\
\text { Vitamin premix }\end{array}$ & 0,100 & 0,100 \\
\hline $\begin{array}{l}\text { Bacitracina de zinco, } 10 \% \\
\text { Zinc bacitracin, } 10 \%\end{array}$ & 0,050 & 0,050 \\
\hline $\begin{array}{l}\text { Coccidiostático coxistac } \\
\text { Coccidiostatic }\end{array}$ & 0,100 & 0,100 \\
\hline $\begin{array}{l}\text { Cloreto de colina, } 60 \% \\
\text { Choline chloride }\end{array}$ & 0,100 & 0,100 \\
\hline $\begin{array}{l}\text { Antioxidante BHT } \\
\text { Antioxidant }\end{array}$ & 0,010 & 0,010 \\
\hline
\end{tabular}

Composição

Composition

\begin{tabular}{lcc}
\hline $\begin{array}{l}\text { Energia metabolizável (kcal/kg) } \\
\text { Metabolizable energy } \\
\text { Proteína bruta, }(\%)^{2}\end{array}$ & 3100 & 3200 \\
$\begin{array}{l}\text { Crude protein } \\
\text { Ca }(\%)^{1}\end{array}$ & 19,80 & 18,50 \\
$\begin{array}{l}\text { Fósforo disponível (\%) } \\
\text { Available phosphorus }\end{array}$ & 0,91 & 0,90 \\
$\begin{array}{l}\text { Metionina (Methionine) }(\%)^{2} \\
\text { Metionina + cistina }(\%)^{2}\end{array}$ & 0,42 & 0,41 \\
$\begin{array}{l}\text { Methionine+cystine } \\
\text { Treonina (\%) }\end{array}$ & 0,48 & 0,43 \\
$\begin{array}{l}\text { Threonine } \\
\text { Arginina (Arginine) }(\%)^{2}\end{array}$ & 0,73 & 0,75 \\
Lisina (Lysine) $(\%)^{1}$ & 1,14 & 0,67 \\
Lisina (Lysine) $(\%)^{2}$ & 0,80 & 1,02 \\
\hline
\end{tabular}

Crescimento (growth): Vit. A, $12.000 .000 \mathrm{UI} \cdot V$ it $\mathrm{D}_{3}, 2.500 .000 \mathrm{Ul} \cdot \mathrm{Vit}$ E, 30.000 Ul; Vit. K, 3 g; Vit. B , 2 g; Vit. B ${ }_{2}, 7$ g; Vit. B 6 , 3 g; Pantotenato de cálcio (Calcium pantotenate), $10 \mathrm{~g}$; Ácido nicotínico (Nicotinic acid), $35 \mathrm{~g}$; Vit. B $12,0,015 \mathrm{~g}$; Biotina (Biotin), 0,07 g; Ácido fólico (Folic acid), $1 \mathrm{~g}$; Selênio (Selenium), 0,12 g; excipiente q.s.q., $500 \mathrm{~g}$.

Aves: Ferro (Iron), 100 g; Manganês (Manganese), 160 g; Zinco (Zinc), $100 \mathrm{~g}$; Cobre (Copper), $20 \mathrm{~g}$; Cobalto (Cobalt), $2 \mathrm{~g}$; lodo (Iodine), $2 \mathrm{~g}$; excipiente q.s.q., $1000 \mathrm{~g}$.

1 Valores calculados de acordo com ROSTAGNO et al. (1983).

2 Valores analisados no Laboratório da Degussa AG. Alemanha.

1 Calculated values with basis on ROSTAGNO et al. (1983).

2 Values analyzed $v$ at the Degussa AG laboratory, Germany. fêmeas da marca Ross e do ganho de peso das fêmeas de ambas as marcas, que não diferiram significativamente $(\mathrm{P}>0,05)$, o ganho de peso e a conversão alimentar das aves apresentaram resposta quadrática aos níveis dietéticos de lisina.

Constata-se, na Tabela 2, que os machos das marcas Ross e Hubbard apresentaram 1265,4 e 1269,2 g de ganho de peso para os níveis de 0,98 e $0,92 \%$ de lisina, respectivamente, e as fêmeas das marcas Ross e Hubbard, 1047,0 e 1017,4 g de ganho de peso, respectivamente, para o nível de $0,98 \%$ de lisina. Para a variável conversão alimentar, os machos Ross e Hubbard apresentaram 1,889 e 1,909 de conversão alimentar, respectivamente, com níveis de $0,98 \%$ de lisina, enquanto as fêmeas Ross e Hubbard, 2,007 e 2,078 de conversão alimentar, respectivamente, com níveis de 0,98 e $1,04 \%$ de lisina na ração. Estes resultados são semelhantes aos obtidos por MORAN JR. e BILGILI (1990), que constataram serem os machos mais eficientes que as fêmeas.

As equações de regressão, com as respectivas exigências estimadas, encontram-se na Tabela 3 . Pode-se inferir que as exigências dos animais da marca Ross foram superiores às da Hubbard em $2,9 \%$. A estimativa de exigência dos machos, considerando-se o ganho de peso e a conversão alimentar, de ambas as marcas, variou de 0,974 a 1,03\% de lisina. Com isso, pode-se estimar a exigência dos machos em 1,03\% de lisina e das fêmeas, em 0,993\% de lisina. Essa estimativa de maior exigência dos machos em relação às fêmeas é coerente com a recomendação de WALDROUP (1991), em que o autor recomenda 0,99 e $0,95 \%$ de lisina para machos e fêmeas, respectivamente. Contudo, ROSTAGNO et al. (1983) e o NRC (1984) desconsideram o efeito de sexo, recomendando, respectivamente, os níveis de 0,95 e $1,0 \%$ de lisina para essa fase de crescimento.

Os resultados de conversão alimentar concordam com os obtidos por MORAN JR. e BILGILI (1990), que, ao suplementarem uma dieta à base de milho, farelo de soja e farelo de gergelim com lisina, atingindo os níveis de 0,$85 ; 0,95$; e $1,05 \%$ de lisina total, verificaram eficiência significativa $(\mathrm{P}<0,05)$ na conversão alimentar, à medida que se elevou o teor de lisina.

MENDES et al. (1997) não observaram efeitos dos níveis de lisina e diferentes relações arginina:lisina estudadas sobre os parâmetros avaliados - como ganho de peso e conversão alimentar - em frangos de corte no período de 22 a 42 dias de idade.

Os resultados, em porcentagem, de rendimento de peito com osso e carne de peito são apresentados 
Tabela 2 - Efeito dos níveis de lisina da ração sobre o desempenho de machos e fêmeas de duas marcas comerciais de frangos de corte no período de 22 a 40 e de 42 a 48 dias de idade

Table 2 - Effect of lysine levels on the performance of males and females of two trade marks of broiler chickens from 22 to 40 and 42 to 48 days of age

Nível de lisina

Level of lysine

\begin{tabular}{|c|c|c|c|c|c|c|c|c|}
\hline & \multicolumn{4}{|c|}{ Ross } & \multicolumn{4}{|c|}{ Hubbard } \\
\hline & \multicolumn{2}{|c|}{$\begin{array}{c}\text { Ganho de peso }(\mathrm{g}) \\
\text { Weight gain }\end{array}$} & \multicolumn{2}{|c|}{$\begin{array}{l}\text { Conversão alimentar } \\
\text { Feed:gain ratio }\end{array}$} & \multicolumn{2}{|c|}{$\begin{array}{c}\text { Ganho de peso }(\mathrm{g}) \\
\text { Weightgain }\end{array}$} & \multicolumn{2}{|c|}{$\begin{array}{l}\text { Conversão alimentar } \\
\text { Feed:gain ratio }\end{array}$} \\
\hline & $\mathrm{M}$ & $\mathrm{F}$ & $\mathrm{M}$ & $\mathrm{F}$ & M & $\mathrm{F}$ & $\mathrm{M}$ & $\mathrm{F}$ \\
\hline & \multicolumn{8}{|c|}{$22-40$ dias (days) } \\
\hline 0,80 & 1155,9 & 1026,1 & 2,035 & 2,112 & 1171,6 & 963,0 & 2,095 & 2,219 \\
\hline 0,86 & 1189,1 & 1026,8 & 1,988 & 2,066 & 1208,6 & 980,3 & 2,026 & 2,143 \\
\hline 0,92 & 1244,6 & 1045,7 & 1,907 & 2,018 & 1269,2 & 999,3 & 1,920 & 2,104 \\
\hline 0,98 & 1265,4 & 1047,0 & 1,889 & 2,007 & 1261,2 & 1017,4 & 1,909 & 2,093 \\
\hline 1,04 & 1237,2 & 1032,4 & 1,892 & 2,011 & 1256,2 & 1008,1 & 1,950 & 2,078 \\
\hline 1,10 & 1236,0 & 1028,4 & 1,900 & 2,015 & 1210,2 & 962,0 & 1,953 & 2,129 \\
\hline Média & 1221,4 & 1034,4 & 1,935 & 2,038 & 1229,5 & 988,4 & 1,976 & 2,128 \\
\hline Mean & & & & & & & & \\
\hline Regressão & $\mathrm{Q}^{*}$ & NS & $\mathrm{Q}^{* *}$ & NS & $\mathrm{Q}^{* *}$ & NS & $\mathrm{Q}^{* *}$ & $\mathrm{Q}^{* *}$ \\
\hline Regression & & & & & & & & \\
\hline \multirow[t]{2}{*}{$\mathrm{CV}(\%)$} & \multicolumn{2}{|c|}{3,73} & \multicolumn{2}{|c|}{2,84} & \multicolumn{2}{|c|}{3,73} & \multicolumn{2}{|c|}{2,84} \\
\hline & \multicolumn{8}{|c|}{$42-48$ dias (days) } \\
\hline 0,75 & 526,5 & 297,5 & 2,176 & 3,070 & 449,5 & 317,8 & 2,565 & 3,097 \\
\hline 0,81 & 531,0 & 322,5 & 2,093 & 2,827 & 510,3 & 322,5 & 2,321 & 2,964 \\
\hline 0,87 & 547,5 & 345,0 & 2,069 & 2,690 & 513,8 & 324,3 & 2,313 & 2,935 \\
\hline 0,93 & 532,5 & 346,8 & 2,132 & 2,728 & 500,0 & 322,0 & 2,316 & 2,882 \\
\hline 0,99 & 529,0 & 332,5 & 2,158 & 2,734 & 500,0 & 318,8 & 2,337 & 2,998 \\
\hline 1,05 & 526,3 & 324,3 & 2,221 & 2,931 & 497,5 & 311,0 & 2,371 & 3,089 \\
\hline Média & 532,1 & 328,1 & 2,142 & 2,830 & 495,2 & 319,4 & 2,371 & 2,994 \\
\hline Mean & & & & & & & & \\
\hline Regressão & NS & NS & NS & NS & NS & NS & NS & NS \\
\hline Regression & & & & & & & & \\
\hline CV $(\%)$ & \multicolumn{2}{|c|}{10,18} & \multicolumn{2}{|c|}{16,37} & \multicolumn{2}{|c|}{10,18} & \multicolumn{2}{|c|}{16,37} \\
\hline
\end{tabular}

$\mathrm{Q}^{* \star}$ - Efeito quadrático $(\mathrm{P}<0,01), \mathrm{Q}^{\star *}$ - Quadratic effect $(P<.01)$.

$Q^{*}$ - Efeito quadrático $(P<0,05), Q^{*}$ - Quadratic effect $(P<.05)$.

NS - Não-significativo - (Not significant).

M - Macho (Male), F - Fêmea (Female).

Tabela 3 - Estimativas das exigências de lisina e regressões para as variáveis ganho de peso e conversão alimentar, considerando o percentual de lisina na dieta para frangos de corte no período de 22 a 40 dias de idade

Table 3 - Estimates of lysine requirements and regressions for weight gain and feed:gain ratio, according to the lysine percent in the diet for broiler chickens from 22 to 40 days of age

\begin{tabular}{|c|c|c|c|}
\hline & $\begin{array}{c}\text { Exigência } \\
\text { Requirement }\end{array}$ & $\begin{array}{l}\text { Regressão } \\
\text { Regression }\end{array}$ & $\mathrm{r}^{2}$ \\
\hline \multicolumn{4}{|c|}{$22-40$ dias (days) } \\
\hline $\begin{array}{l}\text { Ganho de peso } \\
\text { Weight gain }\end{array}$ & & & \\
\hline $\begin{array}{l}\text { Ross macho (Male) } \\
\text { Hubbard macho (Male) }\end{array}$ & $\begin{array}{l}1,004 \\
0,974\end{array}$ & $\begin{array}{l}\hat{Y}=-1274,97+5041,44 X-2511,55 X^{2} \\
\hat{Y}=-1924,84+6551,16 X-3361,75 X^{2}\end{array}$ & $\begin{array}{l}0,97 \\
0,94\end{array}$ \\
\hline \multicolumn{4}{|l|}{$\begin{array}{l}\text { Conversão alimentar } \\
\text { Feed:gain ratio }\end{array}$} \\
\hline $\begin{array}{l}\text { Ross macho (Male) } \\
\text { Hubbard macho (Male) } \\
\text { Hubbard fêmea (Female) }\end{array}$ & $\begin{array}{l}1,030 \\
0,998 \\
0,993\end{array}$ & $\begin{array}{l}\hat{Y}=5,04436-6,1291 X+2,97385 X^{2} \\
\hat{Y}=6,58386-9,35369 X+4,68522 X^{2} \\
\hat{Y}=5,64942-7,18142 X+3,61497 X^{2}\end{array}$ & $\begin{array}{l}0,95 \\
0,92 \\
0,98\end{array}$ \\
\hline
\end{tabular}


Rev. bras. zootec.

na Tabela 4, na qual se constata que não houve efeito dos níveis de lisina sobre o rendimento destas variáveis, exceto para a variável peito com osso dos machos da marca Hubbard, em que houve efeito quadrático $(\mathrm{P}<0,01)$, representado pela equação $\hat{Y}=-19,0643+106,58 X-57,2071 X^{2}, r^{2} 0,78$, a partir da qual se estima exigência de $0,932 \%$ de lisina para máximo rendimento de peito com osso nesse período. Estes resultados confirmam os obtidos por MORAN JR. e BILGILI (1990), quando os autores utilizaram 0,$85 ; 0,95 ;$ e $1,05 \%$ de lisina na ração.

No entanto, apesar de os níveis de lisina não terem apresentado efeito significativo para as variáveis em estudo, pode-se estimar, em termos de valor absoluto, melhor desempenho das aves, para o nível de $0,98 \%$ de lisina na ração, ou seja, quando se compara com o nível de $0,80 \%$ de lisina, houve ganhos da ordem de 3,2 e $7 \%$ para rendimento de peito com osso e carne de peito, respectivamente, sendo que as fêmeas, de ambas as marcas, apresentaram em torno de $3 \%$ a mais no rendimento de peito com osso e peito sem osso, quando comparadas aos machos.

Com relação à variável de rendimento de peito, os resultados são semelhantes aos obtidos por MORAN JR. e BILGILI (1990) e HOLSHEIMER e VEERKAMP (1992), que verificaram efeito quadrático $(\mathrm{P}<0,01)$ sobre essa variável. Esses resultados, de acordo com BAKER e MOLITORIS (1991), são coerentes, uma vez que a lisina tem grande participação na composição da proteína muscular.

Tabela 4 - Efeito dos níveis de lisina sobre o rendimento de peito com osso e carne de peito, em porcentagem, de machos e fêmeas de duas marcas comerciais de frangos de corte no período de 22 a 40 e de 42 a 48 dias de idade

Table 4 - Effect of lysine levels on the breast with bone yield and breast meat yield of males and females of two trade mark of broiler chickens from 22 to 40 and 42 to 48 days of age

Nível de lisina

Level of lysine

\begin{tabular}{|c|c|c|c|c|c|c|c|c|}
\hline & \multicolumn{4}{|c|}{ Ross } & \multicolumn{4}{|c|}{ Hubbard } \\
\hline & \multicolumn{2}{|c|}{$\begin{array}{l}\text { Peito com osso } \\
\text { Breast with bone }\end{array}$} & \multicolumn{2}{|c|}{$\begin{array}{c}\text { Carne de peito } \\
\text { Breast meat }\end{array}$} & \multicolumn{2}{|c|}{$\begin{array}{l}\text { Peito com osso } \\
\text { Breast with bone }\end{array}$} & \multicolumn{2}{|c|}{$\begin{array}{l}\text { Carne de peito } \\
\text { Breast meat }\end{array}$} \\
\hline & M & $\mathrm{F}$ & M & $\mathrm{F}$ & M & $\mathrm{F}$ & M & $\mathrm{F}$ \\
\hline & \multicolumn{8}{|c|}{22 - 40 dias (days) } \\
\hline 0,80 & 29,6 & 31,3 & 19,6 & 20,9 & 29,5 & 29,5 & 19,4 & 19,4 \\
\hline 0,86 & 30,1 & 31,6 & 20,5 & 21,3 & 30,5 & 30,2 & 20,2 & 20,1 \\
\hline 0,92 & 30,1 & 31,7 & 20,6 & 21,4 & 30,6 & 30,3 & 20,4 & 20,4 \\
\hline 0,98 & 30,9 & 31,9 & 21,6 & 21,8 & 30,7 & 30,4 & 20,6 & 20,8 \\
\hline 1,04 & 31,1 & 31,9 & 21,0 & 21,8 & 29,3 & 30,4 & 19,5 & 20,7 \\
\hline 1,10 & 30,9 & 31,7 & 20,6 & 21,8 & 29,2 & 30,3 & 19,4 & 20,5 \\
\hline Média & 30,5 & 31,7 & 20,7 & 21,5 & 30,0 & 30,2 & 19,9 & 20,3 \\
\hline $\begin{array}{l}\text { Mean } \\
\text { Regressão } \\
\text { Regression }\end{array}$ & NS & NS & NS & NS & $\mathrm{Q}^{* *}$ & NS & NS & NS \\
\hline \multirow[t]{2}{*}{$\mathrm{CV}(\%)$} & \multicolumn{2}{|c|}{2,82} & \multicolumn{2}{|c|}{4,32} & \multicolumn{2}{|c|}{2,82} & \multicolumn{2}{|c|}{4,32} \\
\hline & \multicolumn{8}{|c|}{$42-48$ dias (days) } \\
\hline 0,75 & 30,2 & 30,9 & 19,7 & 20,5 & 29,6 & 29,9 & 18,6 & 18,2 \\
\hline 0,81 & 30,7 & 30,7 & 19,1 & 20,2 & 30,3 & 31,7 & 18,3 & 18,3 \\
\hline 0,87 & 30,9 & 31,8 & 20,1 & 21,3 & 30,3 & 31,7 & 18,8 & 19,7 \\
\hline 0,93 & 30,6 & 31,9 & 19,3 & 20,7 & 30,5 & 30,7 & 18,9 & 19,3 \\
\hline 0,99 & 30,7 & 31,3 & 19,9 & 20,6 & 30,1 & 30,2 & 19,5 & 18,7 \\
\hline 1,05 & 30,5 & 31,3 & 19,9 & 20,6 & 29,4 & 30,6 & 19,3 & 19,4 \\
\hline Média & 30,6 & 31,3 & 19,7 & 20,7 & 30,0 & 30,8 & 18,9 & 18,9 \\
\hline Mean & & & & & & & & \\
\hline Regressão & NS & NS & NS & NS & NS & $\mathrm{Q}^{*}$ & NS & NS \\
\hline Regression & & & & & & & & \\
\hline CV $(\%)$ & \multicolumn{2}{|c|}{2,72} & \multicolumn{2}{|c|}{4,92} & \multicolumn{2}{|c|}{2,72} & \multicolumn{2}{|c|}{4,92} \\
\hline
\end{tabular}

$\mathrm{Q}^{\star \star}$ - Efeito quadrático $(P<0,01), \mathrm{Q}^{\star \star}-$ Quadratic effect $(P<.01)$.

$\mathrm{Q}^{*}$ - Efeito quadrático $(\mathrm{P}<0,05), \mathrm{Q}^{*}$ - Quadratic effect $(P<.05)$.

NS - Não-significativo - (Not significant).

M - Macho (Male), F - Fêmea (Female). 
HICKLING et al. (1989) constataram incremento de $2,8 \%$ no rendimento de peito com níveis crescentes de lisina (0,95 a 1,15\% de lisina). No presente trabalho, para variação de 0,8 a $0,98 \%$ de lisina, a melhora foi de $3,9 \%$ no rendimento de peito.

Tomando como base os melhores resultados, em valores absolutos, do ganho de peso, da conversão alimentar, dos rendimentos de peito com osso e carne de peito de machos e fêmeas, de ambas as marcas, pode-se observar que os valores de exigências variaram de 0,87 a $0,93 \%$ de lisina.

Níveis de lisina da ração no desempenho de frangos de corte no período de 42 a 48 dias de idade

Não houve mortalidade durante o período experimental; os resultados de desempenho das aves, machos e fêmeas, estão apresentados na Tabela 2. Não houve efeito significativo $(P>0,05)$ dos níveis de lisina sobre consumo de ração, ganho de peso e conversão alimentar das aves. Entretanto, observouse melhor desempenho para ambos os sexos das duas marcas, com o nível de $0,87 \%$ de lisina, exceto para as fêmeas Hubbard, que apresentaram conversão alimentar mais eficiente, com $0,93 \%$ de lisina na ração.

Analisando-se os resultados médios das variáveis ganho de peso e conversão alimentar, verifica-se que os machos e as fêmeas apresentaram ganhos de 530,6 e $334,4 \mathrm{~g}$, respectivamente, e ambos foram maiores $8,7 \%$ em relação ao ganho de peso obtido com $0,75 \%$ de lisina na ração. Estes resultados são superiores ao valor de $3,7 \%$, obtido por BILGILI et al. (1992), quando o nível de lisina na ração variou de 0,85 a $0,95 \%$. No entanto, para a conversão alimentar, os machos e as fêmeas apresentaram melhoras de 7,6 e $10,7 \%$, respectivamente, em relação à conversão alimentar, obtida com $0,75 \%$ de lisina na ração.

A exigência nutricional média de $0,87 \%$ de lisina foi maior que a observada por RESENDE et al. (1980b), em que os autores obtiveram exigência de $0,85 \%$ de lisina para máximo ganho de peso, sendo que os machos apresentaram melhor desempenho que as fêmeas. Entretanto, considerando-se a média das exigências de lisina das fêmeas, para o parâmetro conversão alimentar, obtém-se a exigência de $0,93 \%$ de lisina, o que confirma o mesmo resultado obtido por RESENDE et al. (1980b).

Os resultados, em porcentagem, de peito com osso e carne de peito são apresentados na Tabela 4, na qual se constata que não houve efeito dos níveis de lisina sobre o rendimento destes cortes, exceto para a variável peito com osso das fêmeas da marca Hubbard, em que se verificou efeito quadrático $(\mathrm{P}<0,05)$, representado pela equação $\hat{Y}=-3,68818+78,5279 X-$ $44,1319 X^{2} r^{2}=0,35$, a partir da qual se estima exigência de $0,89 \%$ de lisina na ração, para máximo rendimento de carne de peito com osso, nesse período. O resultado confirma os obtidos por BILGILI et al. (1992), os quais verificaram exigência média de 0,85 a $0,95 \%$ de lisina, para máxima deposição de carne de peito. No entanto, embora os níveis de lisina não tenham apresentado efeito significativo, para as variáveis em estudo, pode-se estimar, em termos de valores absolutos, o melhor desempenho das aves para o nível de $0,93 \%$ de lisina na ração.

Considerando-se os melhores resultados, em valores absolutos, do ganho de peso, da conversão alimentar e do rendimento de peito com osso e carne de peito de machos e fêmeas de ambas as marcas, pode-se observar que os valores de exigências variaram de 0,81 a $0,99 \%$ de lisina, resultando em exigência nutricional média de $0,90 \%$ de lisina total na ração; este valor está próximo da estimativa de $0,89 \%$ de lisina para máxima deposição de carne de peito com osso obtida com as fêmeas Hubbard.

\section{Conclusões}

Considerando-se os dados de ganho de peso, conversão alimentar e rendimentos de peito com osso e carne de peito, nos dois experimentos realizados, pode-se recomendar para frangos de corte no período de 22 a 40 dias de idade o nível nutricional de lisina total em $0,993 \%$ para as fêmeas e $1,03 \%$ para os machos, em rações com $3100 \mathrm{kcal} \mathrm{EM/kg}$. Para o período de 42 a 48 dias de idade, recomenda-se o nível de $0,90 \%$ de lisina total, para ambos os sexos, em rações com $3200 \mathrm{kcal} \mathrm{EM/kg}$.

\section{Referências Bibliográficas}

ACAR, N., MORAN JR., E.T., BILGILI, S.F. 1991. Live performance and carcass yield of male broilers from two commercial strain crosses receiving rations containing lysine below and above the established requirement between 6 and 8 weeks of age. Poult. Sci., 70:2315-2321.

AEC - Tables AEC - 1987. Recomendações para a nutrição animal. 5.ed. Ed. Rhône-Poulenc. Commentary - França. 86p.

AL-NASSER, A.Y., ILIAN-AWADI, A.A., DIAB, M.F. 1986. The effect of adding essential amino acids and vitamins to ration of broilers. Poult. Sci., 65:742-748.

AGRICULTURAL RESEARCH COUNCIL - ARC 1975. The nutrient requirements of farm livestock. n. 1 Poultry. London. 154p.

BAKER, D.H., MOLITORIS, B.A. 1991. Partioning of nutrients for growth and other metabolic functions: Efficiency and priority considerations. Poult. Sci., 70:1797-1805. 
Rev. bras. zootec.

BILGILI, S.F., MORAN JR., E.T., ACAR, N. 1992. Strain cross response of heavy male broilers to dietary lysine in the finisher feed: live performance and further - processing yields. Poult. Sci., 71:850-858.

BRAGA, J.M. 1983. Avaliação da fertilidade do solo (ensaios de campo). Viçosa: UFV, Imprensa Universitária. 101p.

DUDLEY-CASH, W.A. 1992. Latest research findings reportd at annual poultry science meeting. Feedstuffs, 64:37.

EUCLYDES, R.F. 1983. Manual de utilização do programa SAEG (Sistema de Análise Estatística e Genética). Viçosa, MG: UFV. 59p.

HICKLING, D., GUENTER, W., JACKSON, M.E. 1989. The effects of dietary lysine and methionine on broiler breast meat yield at different ages. Poult. Sci., 68(1)163-168 (Supplement).

HICKLING, D., GUENTER, W., JACKSON, M.E. 1990. The effects of dietary methionine and lysine on broiler chicken performance and breast meat yield. Can. J. Anim. Sci., 70:673-678.

HOLSHEIMER, J.P., VEERKAMP, C.H. 1992. Effect of dietary energy and lysine content on performance and yields of two strains of males broiler chicks. Poult. Sci., 71:872-879.

INSTITUT NATIONAL DE LA RECHERCHE AGRONOMIQUE INRA. 1984. L'alimentacion des animaux monogastriques. Paris. 279p.

JENSEN, L.S., WYATT,C.L., FANCHER, B.I. 1989. Sulfur amino acid requirement of broiler chickens from 3 to 6 weeks of age. Poult. Sci., 68:163-168.

MENDES, A.A., WATKINS, S.E., ENGLAND, J.A. et al. 1997. Influence of dietary lysine levels and arginine:lysine ratios on performance of broilers exposed to heat or cold stress during the period of three to six weeks of age. Poult. Sci., 76:472-481.

MORAN JR., E.T., BILGILI, S.F. 1990. Processing losses, carcass quality and meat yields for broiler chicken, receiving diets marginally deficient to adequate in lysine prior to marketing. Poult. Sci., 69:702-710.
NATIONAL RESEARCH COUNCIL - NRC. 1984. Nutrient requirements of poultry. 8.ed. Washington, D.C.: National Academic Press. 71p.

NATIONAL RESEARCH COUNCIL - NRC. 1994. Nutrient requirements of poultry. 9.ed. Washington, D.C.: National Academic Press. 155p.

RESENDE, J.A.A., ROSTAGNO, H.S., SILVA, M.A et al. 1980a. Níveis de proteína, aminoácidos sulfurosos e lisina em rações de frangos submetidos a regime de alta temperatura. Fase de crescimento. R. Soc. Bras. Zootec., 9(1):108-124.

RESENDE, J.A.A., ROSTAGNO, H.S., SILVA, M.A et al. 1980b. Níveis de proteína, aminoácidos sulfurosos e lisina em rações de frangos submetidos a regime de alta temperatura. Fase final. R. Soc. Bras. Zootec., 9(1):125-141.

ROSTAGNO, H.S., SILVA, D.J., COSTA, P.M.A. et al. 1983. Composição de alimentos e exigências nutricionais de aves e suínos. Viçosa, UFV. 61p (Tabelas brasileiras).

ROSTAGNO, H.S. Valores de composição de alimentos e de exigências nutricionais utilizados na formulação de rações para aves. In: REUNIÃO ANUAL DA SOCIEDADE BRASILEIRA DEZOOTECNIA. Piracicaba, 1990. Anais... Piracicaba: FEALQ. 1990. p.11-30.

SCOTT, M.L., NESHEIM, M.C., YOUNG, R.J. 1982. Nutrition of chickens. 5.ed. Ithaca. 562p.

WALDROUP, P.W. 1991. Dietary nutrients allowances for chickens and turkeys. Feedstuffs, 63:29.

Recebido em: 03/08/1999

Aceito em: 08/02/2000 